\title{
Pneumothorax and subcutaneous emphysema secondary to blunt chest injury
}

\author{
Jahan Porhomayon ${ }^{{ }^{*}}$, Ralph Doerr ${ }^{2}$
}

\section{Abstract}

This is the case of a patient with a history of blunt chest trauma associated with subcutaneous emphysema and pneumothorax. The patient complained of inspiratory stridor on presentation. Anatomical relationships can explain the pathophysiological process.

\section{Case report}

A 49-year-old male presented to the trauma service $10 \mathrm{~h}$ after blunt chest injury. Initial presentation included respiratory failure with a respiratory rate of 26 beats per minute, a pulse rate of 110 beats per minute, and blood pressure of 150/80 mmHg. He complained of dysphonia and facial swelling.

Physical examination revealed inspiratory dyspnea and crepitations suggestive of subcutaneous emphysema of the face, neck, and upper portion of his chest. Pharyngeal examination revealed swollen mucosa with crepitations on palpation (Figure 1a). Chest X-ray indicated extensive subcutaneous emphysema apparent in part as a group of muscles in the upper chest wall, but with no obvious pneumothorax (Figure 1c). Computed tomography of the chest confirmed subcutaneous and submucosal emphysema involving the pharynx. It also revealed obvious pneumomediastinum associated with left pneumothorax from rib fractures (Figure $1 \mathrm{~b}$ and $1 \mathrm{~d}$ ). Physical examination and bronchoscopy ruled out laryngotracheal mucosal rupture. The patient remained dyspneic after placement of a chest tube. Twenty-four hours later, inspiratory dyspnea, dysphonia, and submucosal emphysema had resolved. Subcutaneous emphysema resolved in 4 days. The patient's recovery was uneventful.

\footnotetext{
* Correspondence: jahanpor@buffalo.edu

'VA Western New York Healthcare System, Division of Critical Care and Pain Medicine, Department of Anesthesiology, State University of New York at Buffalo School of Medicine and Biomedical Sciences, VA Medical Center, Rm 203C, 3495 Bailey Ave, 14215, Buffalo, New York, USA

Full list of author information is available at the end of the article
}

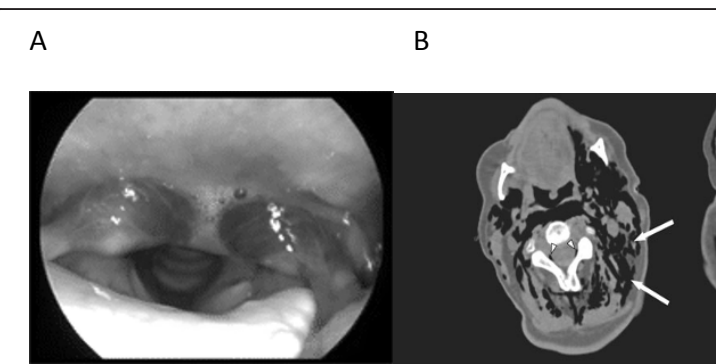

C

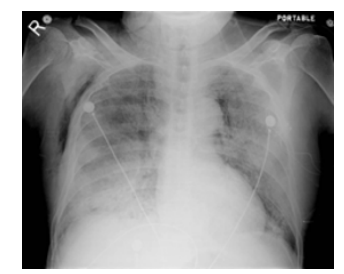

D

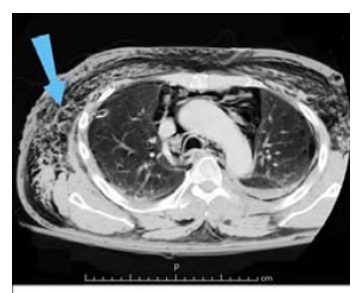

Figure 1 Subcuatneous and Submucosal emphysema. A) Pharyngeal submucosal emphysema B) CT image with subcutaneous air $\mathbf{C}$ ) The chest radiograph shows extensive subcutaneous emphysema D) The CT-scan shows pneumothorax, pneumomediastinum and subcutaneous emphysema.

\section{SpringerOpen ${ }^{\circ}$}

C 2011 Porhomayon and Doerr; licensee Springer. This is an Open Access article distributed under the terms of the Creative Commons Attribution License (http://creativecommons.org/licenses/by/2.0), which permits unrestricted use, distribution, and reproduction in any medium, provided the original work is properly cited. 

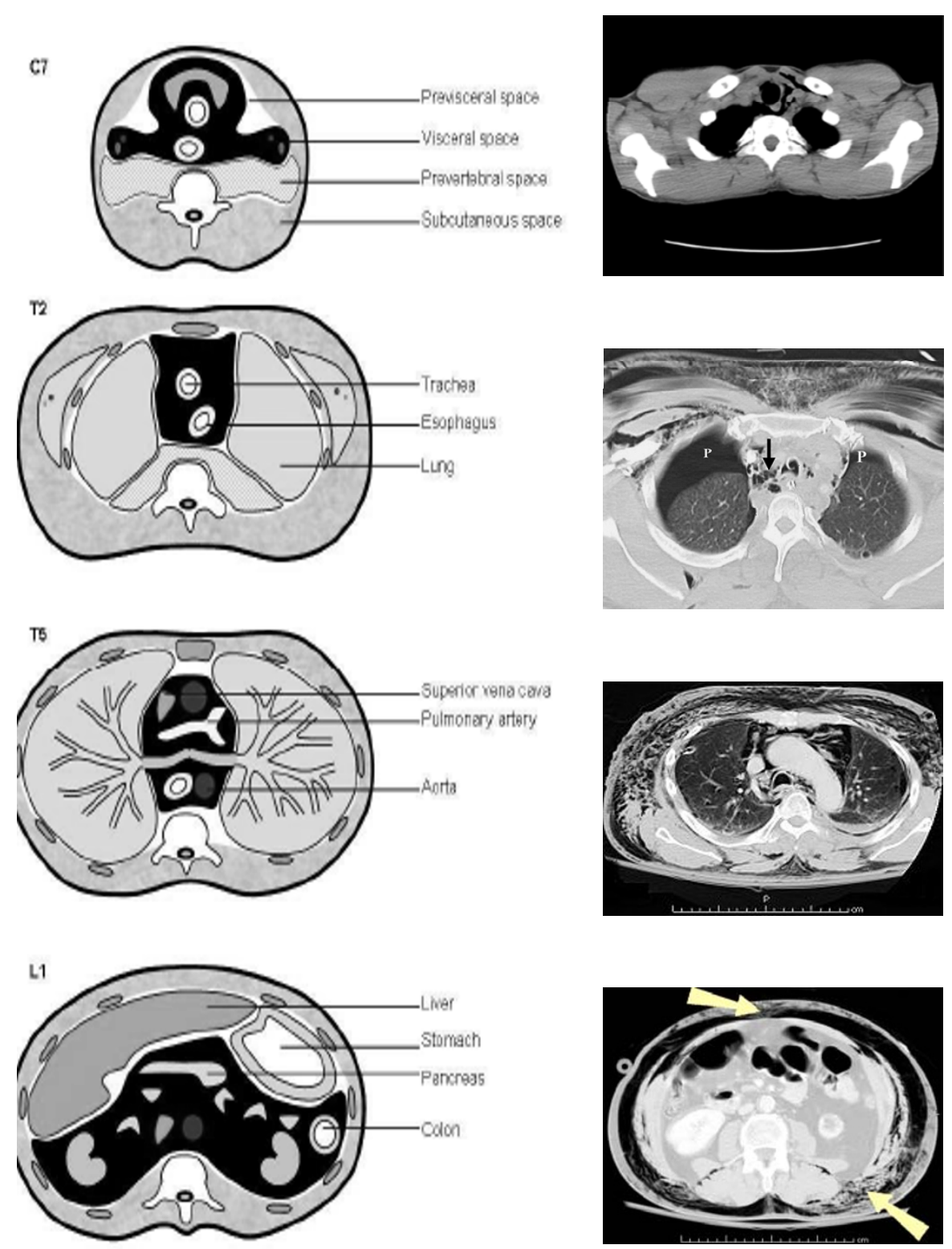

Figure 2 Anatomical relationship of abdominal, thoracic and cervical fascial planes. A) Anatomical relationship of the cervical and thoraco-abdominal region B) Air can diffuse through cervical, mediastinal and retroperitoneal region.

\section{Discussion}

Subcutaneous emphysema can occur in critically ill patients after blunt trauma to the chest and result in a pressure gradient between the intra-alveolar and perivascular interstitial space $[1,2]$. The chest radiograph cannot exclude pneumothorax or pneumomediastinum. A CT scan is often needed for assessment of these conditions. Oropharyngeal subcutaneous emphysema has been described with dental surgery or spontaneous rupture of oropharyngeal or bronchial mucosa $[3,4]$. The association of submucosal emphysema with pneumothorax is rare.
However, anatomical correlation among fascial planes of the cervical area, mediastinum, and retroperitoneum can explain this relationship [1] (Figure 2a and 2b).

\section{Acknowledgements}

The authors obtained permission from the patient to display images and photographs in scientific journals.

\section{Author details}

'VA Western New York Healthcare System, Division of Critical Care and Pain Medicine, Department of Anesthesiology, State University of New York at Buffalo School of Medicine and Biomedical Sciences, VA Medical Center, Rm 
203C, 3495 Bailey Ave, 14215, Buffalo, New York, USA Via Health of New York Health Care System, Rochester, New York, USA; State University of New York at Buffalo School of Medicine and Biomedical Sciences, 3495 Bailey Ave, 14215 Buffalo, New York, USA

\section{Authors' contributions}

Both authors contributed to writing the manuscript.

\section{Competing interests}

The authors do not have any financial and personal relationships with other people or organizations that could inappropriately influence (bias) their work. Examples of potential conflicts of interest include employment, consultancies, stock ownership, honoraria, paid expert testimony, patent applications/registrations, and grants or other funding.

Received: 9 April 2010 Accepted: 21 March 2011

Published: 21 March 2011

\section{References}

1. Maunder RJ, Pierson DJ, Hudson LD: Subcutaneous emphysema. Pathophysiology, diagnosis, and management. Arch Intern Med 1984, 144(7):1447-53.

2. Wintermark M, Schnyder P: The Macklin effect: a frequent etiology for pneumomediastinum in severe blunt chest trauma. Chest 2001, 120(2):543-7.

3. Larsen KD: Submucosal emphysema with airway obstruction from nasal oxygen cannula. Anesth Analg 1988, 67(6):586-7.

4. Sacco JJ, Halliday DW: Submucosal epiglottic emphysema complicating bronchial rupture. Anesthesiology 1987, 66(4):555-7.

doi:10.1186/1865-1380-4-10

Cite this article as: Porhomayon and Doerr: Pneumothorax and subcutaneous emphysema secondary to blunt chest injury. International Journal of Emergency Medicine 2011 4:10.

\section{Submit your manuscript to a SpringerOpen ${ }^{\mathcal{O}}$ journal and benefit from:}

- Convenient online submission

- Rigorous peer review

- Immediate publication on acceptance

- Open access: articles freely available online

- High visibility within the field

- Retaining the copyright to your article

Submit your next manuscript at $\gg$ springeropen.com 